\title{
Multimodal encoding in a cortical model for spatial navigation planning Louis-Emmanuel Martinet ${ }^{* 1,2,3}$, Denis Sheynikhovich ${ }^{1,2}$, Jean-Arcady Meyer ${ }^{3}$ and Angelo Arleo ${ }^{1,2}$
}

Address: ${ }^{1}$ UPMC Univ Paris 6, UMR 7102, F-75005, Paris, France, ${ }^{2}$ CNRS, UMR 7102, F-75005, Paris, France and ${ }^{3}$ UPMC Univ Paris 6, UMR 7222 , ISIR, F-75005, Paris, France

Email: Louis-Emmanuel Martinet* - louis-emmanuel.martinet@upmc.fr

* Corresponding author

from Eighteenth Annual Computational Neuroscience Meeting: CNS*2009

Berlin, Germany. 18-23 July 2009

Published: 13 July 2009

BMC Neuroscience 2009, I0(Suppl I):P338 doi:I0.II86/I47|-2202-I0-SI-P338

This abstract is available from: http://www.biomedcentral.com/I47/-2202/I0/SI/P338

(c) 2009 Martinet et al; licensee BioMed Central Ltd.

According to experimental evidence, spatial navigation planning is likely to rely upon a distributed neural network spanning limbic and cortical brain structures. This network includes (i) the hippocampus, which mediates robust spatial representations, and (ii) neocortical structures, such as the prefrontal cortex, which participate in the elaboration of more abstract contextual descriptions (e.g., accounting for motivation-dependent memories and action cost/risk constraints). In order to investigate this working hypothesis, we model the interaction between the hippocampus [1] and the prefrontal cortex [2]. We focus on the cortical columnar organization to study a neuromimetic architecture suitable for spatial navigation planning. We validate the system's learning performance on a classical spatial behavioural task, the Tolman \& Honzik's detour protocol [3], which suggests that rodents can plan flexible goal-directed trajectories in the presence of blocked pathways. We also put forth a set of statistical analyses to assess the spatial coding properties of the model hippocampal place and cortical column cells.

Here, we couple our hippocampal place cell [1] and columnar cortical [2] models to provide a better understanding of the dynamics of the action planning neural network. We also improve the biological plausibility of the cortical model, by explicitly identifying the subpopulations of (rate code) neurones that encode different information (e.g., current spatial state, goal-related and prospective memory signals, local actions). This approach has several advantages: (i) the response of each subpopulation being more specific, it makes it possible to perform a series of analyses of multiple neural activity correlates; (ii) the functioning of the columnar assembly can be formalised within the reinforcement learning framework, which proved to be relevant to the understanding of goalrelated neural activities [4]; (iii) the biological plausibility of the model being enhanced, the discharges of formal units can be compared against experimental data (e.g., electrophysiological recordings $[5,6])$, and give rise to testable predictions.

The spatial planning model reproduces the experimental results by Tolman \& Honzik [3]. It also unravels the possible links between the single unit level and the behavioural level relevant to the learning of the task (e.g., to the selection of the shortest path to the reward, and to the prediction of future state sequences). Finally, our neural response analysis suggests how the interplay between the model hippocampus and the prefrontal cortex can yield to the encoding of manifold information pertinent to the spatial planning function (e.g., prospective and distanceto-goal correlates). 


\section{Acknowledgements}

Granted by EC Project ICEA (Integrating Cognition, Emotion and Autonomy), IST-027819-IP.

\section{References}

I. Sheynikhovich D, Chavarriaga R, Stroesslin T, Arleo A, Gerstner W: Is there a geometric module for spatial orientation? Insights from a rodent navigation model. . Submitted

2. Martinet LE, Passot JB, Fouque B, Meyer JA, Arleo A: Map-based spatial navigation: A cortical column model for action planning. Spatial Cognition VI: LNAI 5248 2008:39-55.

3. Tolman EC, Honzik CH: "Insight" in rats. Univ Calif Publ Psychol 1930, 4:215-232.

4. Schultz W, Dayan P, Montague PR: A neural substrate of prediction and reward. Science 1997, 275:1593-1599.

5. Hok V, Save E, Lenck-Santini PP, Poucet B: Coding for spatial goals in the prelimbic/infralimbic area of the rat frontal cortex. PNAS 2005, 102:4602-4607.

6. Benchenane K, Peyrache A, Khamassi M, Battaglia FP, Wiener SI: Coherence of theta rhythm between hippocampus and medial prefrontal cortex modulates prefrontal network activity during learning in rats. Society for Neuroscience Meeting 2008.

Publish with Bio Med Central and every scientist can read your work free of charge

"BioMed Central will be the most significant development for disseminating the results of biomedical research in our lifetime. "

Sir Paul Nurse, Cancer Research UK

Your research papers will be:

- available free of charge to the entire biomedical community

- peer reviewed and published immediately upon acceptance

- cited in PubMed and archived on PubMed Central

- yours - you keep the copyright

Submit your manuscript here:

http://www.biomedcentral.com/info/publishing_adv.asp 